\title{
Morfologia polínica de Camarea St.-Hil. (Malpighiaceae) ${ }^{1}$
}

\author{
HIROKO MAKINO-WATANABE 2 , THEREZINHA SANT'ANNA MELHEM ${ }^{2,4} \mathrm{e}$ \\ ORTRUD MONIKA BARTH ${ }^{3}$
}

(recebido em 24/01/96; aceito em 16/05/97)

\begin{abstract}
Pollen grain morphology of Camarea St.-Hil. (Malpighiaceae)). Pollen grains of eight species of Camarea, including a hybrid one are described and illustrated. The species present very similar pollen grains showing some differences in the apertures and ornamentation type. An identification key of the species studied is also presented.
\end{abstract}

RFSUMO - (Morfologia polínica de Camarea St.-Hil. (Malpighiaceae)). Grãos de pólen de oito espécies de Camarea, incluindo uma espécie híbrida, são descritos e ilustrados. As espécies apresentam grãos de pólen muito semelhantes mostrando algumas diferenças nas aberturas e tipo de ornamentação. Uma chave de identificação das espécies estudadas é também apresentada.

Key words - Pollen grains, Camarea, Malpighiaceae

\section{Introdução}

O gênero Camarea St.-Hil. é constituído de espécies subarbustivas, com flores amarelas vistosas, ocorrendo especialmente nos cerrados e campos rupestres, sendo o planalto central brasileiro seu centro de diversidade (Mamede 1990a).

De acordo com a recente revisão feita por Mamede (1990a), Camarea pertence à tribo Gaudichaudieae sensu Jussieu e compreende sete espécies $C$. affinis St.-Hil., C. axillaris St.-Hil., $C$. elongata Mamede, $C$. ericoides St.-Hil., C. hirsuta St.-Hil., C. linearifolia St.-Hil. e C. sericea St.-Hil.) e um provável híbrido entre $C$. affinis St.-Hil. x C. hirsuta St.-Hil.

Poucos são os estudos palinológicos no gênero, podendo-se citar os trabalhos de Erdtman (1971) e Salgado-Labouriau (1973), os quais fazem referência aos grãos de pólen de $C$. ericoides e $C$. triphylla.

O presente estudo é mais uma contribuição à palinotaxonomia do gênero Camarea.

\section{Material e métodos}

Os materiais utilizados foram obtidos a partir de exsicatas depositadas em vários herbários brasileiros (HB, R, RB, SP, UB, UEC). Sempre que possível procurou-se estudar vários espécimes de uma mesma espécie sendo um deles tomado como material

1. Parte da tese de doutorado de H.M. Watanabe.

2. Instituto de Botânica, Caixa Postal 4005, 01061-970 São Paulo, SP, Brasil,

3. Instituto Oswaldo Cruz, Caixa Postal 906, 20001-970 Rio de Janeiro, RJ, Brasil.

4. Bolsista do CNPq. padrão (assinalado no material examinado por um asterisco), no qual foram efetuadas todas as medidas e observações necessárias para a caracterização dos grãos de pólen. Os demais espécimes foram utilizados como materiais de comparação com o padrão. Material examinado: Camarea affinis St.-Hil. Minas Gerais: Belo Horizonte, 11-I- 1965, W. Handro 38 (SP); Paraopeba, 14-I-1965, W. Handro 82 (SP)*; São João Del-Rey, Serra do Lenheiro, I1960, A.P. Duarte 5158 (HB); São Paulo: São Paulo, Moóca, 23II-1913, A.C. Brade 5315 (SP); São Paulo, Jabaquara, 25-I-1949, O. Handro 64 (SP); C. affinis x C. hirsuta. St.-Hil. Goiás: Monte Alegre, 11-III-1973, William R. Anderson 6849 (UB); Serra Geral do Paraná, São João da Aliança, 25-III-1973, W.R. Anderson 7948 (UB)*; C. axillaris St.-Hil. Bahia: Rio de Contas, 27-X1988, R.M. Harley et al. 25687 (SP)*; Minas Gerais: Diamantina, 05-VI 1985, Maria Amélia V. da Cruz 87(SP); C. elongata Mamede. Bahia: Morro do Chapéu, 3-III-1977, R.M. Harley 19361 (RB)*; 27-X-1978, G. Martinelli 5244 et al. (RB); C. ericoides. St.-Hil. Goiás: Pirenópolis, 22-VII-1952, A. Macedo 3595 (SP); Serra dos Cristais, ca. $2 \mathrm{~km} \mathrm{~N}$ de Cristalina, 5-XI-1965, H.S. Irwin et al. 9919 (HB); Minas Gerais: Belo Horizonte, Morro das Pedras, 18-IX-1945, L.O. William \& Vicente Assis 7571 (R); Diamantina, 17-XI-1937, Mello Barreto 9821 (R); Várzea da Palma, 4-X-1965, M.E.R. Matos et al. 60 (SP)*; C. hirsuta St.-Hil. Minas Gerais: sem indicação precisa de local, coletor e data de coleta (RB62812, Herb. Schwacke 3305); C. linearifolia St.-Hil. Minas Gerais: Itutinga, rod. Lavras-S.J. Del Rey, 10-XII-1980, H.F. Leitão-Filho et al.11894 (UEC); C. sericea St.-Hil. Goiás: Serra Dourada, 8-IX-1976, P. Gibbs et al. 2763 (UEC).

Os materiais polínicos foram preparados pelo método de acetólise (Erdtman 1960).

Tratamentos estatísticos das medidas dos diâmetros de 25 grãos de pólen tomados aleatoriamente foram efetuados, calculando-se a média aritmética $(\overline{\mathrm{x}})$, o desvio padrão da média $\left(\mathrm{s}_{\overline{\mathrm{x}}}\right), \mathrm{o}$ desvio padrão da amostra (s) e o coeficiente de variabilidade (V). Comparações entre as médias foram realizadas, analisando-se o intervalo de confiança (IC) a 95\%. Para as medidas dos demais caracteres como aberturas e parede dos grãos de pólen, bem como para os diâmetros dos materiais de comparação, foi calculada somente a média aritmética de dez medidas.

As fotomicrografias foram obtidas em fotomicroscópio Olympus-Vanox e as elétron-micrografias em microscópio eletrônico de varredura JEOL, modelo JSM-255-II, seguindo-se as etapas citadas em Makino-Watanabe (1988). 


\section{Resultados}

O gênero Camarea é caracterizado por grãos de pólen de tamanho grande (entre 50 e $100 \mu \mathrm{m}$ ) contorno aproximadamente circular a quadrangular, apolares, 6 a 8 poros ladeados por margem fina, sendo mais freqüentes os grãos de pólen com 6 poros. Poros de diferentes tamanhos, em geral associados a colpóides largos e ornamentados $(C$. affinis x $C$ hirsuta, C. axillaris, C. elongata, $C$. ericoides, $C$. hirsuta, $C$. linearifolia) ou estreitos e não esculturados ( $C$. affinis e $C$. sericea), com contornos irregulares (figuras 1 a 20). Nos grãos de pólen com 6 poros, estes estão distribuídos 3 a 3 em planos diferentes, unidos aos colpóides (figuras 3, 13, 16 e 19). Exina tectada, com nexina espessa, principalmente nas regiões próximas às aberturas. Ornamentação da exina variável podendo ser psilada ( $C$ sericea), microrreticulada $(C$. affinis, C. hirsuta, C. affinis x C. hirsuta, $C$. axillaris, $C$. elongata) ou levemente rugulada $(C$. ericoides, $C$. linearifolia) entre os colpóides e granulosa na superfície dos mesmos (figuras 5, 11, 12,16 e 20). As figuras 10 e 11 mostram, em microscopia eletrônica de varredura, o grão de pólen de $C$. ericoides, o qual representa o tipo polínico mais freqüente em Camarea. As demais espécies possuem grãos de pólen que apresentam pequenas variações deste tipo padrão.

O provável híbrido C. affinis x C. hirsuta (figuras 19 e 20) exibe grãos de pólen semelhantes aos das espécies parentais porém com caracteres mais próximos aos de $C$. hirsuta no que se refere às aberturas e largura dos colpóides. $C$. affinis apresenta colpóides finos $(\overline{\mathrm{x}}=1,8 \mu \mathrm{m})$, ao contrário de $C$. affinis $\mathrm{x}$ $C$. hirsuta e $C$. hirsuta, que possuem colpóides largos $(\overline{\mathrm{x}}=8,5 \mu \mathrm{m}$ e $\overline{\mathrm{x}}=7,3 \mu \mathrm{m}$, respectivamente).

Uma característica importante é a presença de arestas salientes, lisas e contínuas, observada em $C$. axillaris (figura 5), C. ericoides (figura 10) e $C$. linearifolia (figura 15) ou descontínuas em $C$. elongata (figura 8), que delimitam faces quadran-

Tabela 1. Medidas dos diâmetros dos grãos de pólen de espécies de Camarea.

\begin{tabular}{llcccc}
\hline Espécies & $\mathrm{FV}(\mu \mathrm{m})$ & $\overline{\mathrm{x}} \pm \mathrm{s}_{\overline{\mathrm{x}}}(\mu \mathrm{m})$ & $\mathrm{s}(\mu \mathrm{m})$ & $\mathrm{V}(\%)$ & $\mathrm{IC}(\mu \mathrm{m})$ \\
\hline C. affinis & $63,8-75,6$ & $69,82 \pm 0,5$ & 2,7 & 3,9 & $68,8-70,8$ \\
C. axillaris & $59,4-69,5$ & $65,5 \pm 0,5$ & 2,7 & 4,1 & $64,5-66,5$ \\
C. elongata & $61,6-72,2$ & $67,7 \pm 0,6$ & 3,0 & 4,4 & $66,5-68,9$ \\
C. ericoides & $61,0-75,8$ & $67,8 \pm 0,7$ & 3,4 & 5,0 & $66,4-69,2$ \\
C. hirsuta & $60,1-67,7 *$ & $\mathrm{x}=63,3$ & - & - & - \\
C. linearifolia & $57,0-67,9$ & $63,3 \pm 0,5$ & 2,7 & 4,3 & $62,3-64,3$ \\
C. sericea & $43,9-54,7$ & $52,1 \pm 0,7$ & 3,6 & 6,9 & $50,7-53,5$ \\
C. affinis x C. hirsuta & $63,3-73,6$ & $69,0+0,5$ & 2,5 & 3,6 & $68,0-70,0$ \\
\hline
\end{tabular}

* Valores baseados em 10 medidas; FV = faixa de variação.

Tabela 2. Medidas ( $\mu \mathrm{m})$ do poro e camadas da exina dos grãos de pólen de Camarea.

\begin{tabular}{|c|c|c|c|c|c|c|c|c|c|c|c|c|}
\hline \multirow{2}{*}{ Espécies } & \multicolumn{4}{|c|}{ Números e medidas do poro* } & \multicolumn{4}{|c|}{ Exina região do poro } & \multicolumn{4}{|c|}{ Exina região do mesoporo } \\
\hline & $\mathrm{n}^{\mathrm{o}}$ & $\mathrm{C}$ & $\mathrm{L}$ & M & Exina & Sexina & Nexina 1 & Nexina 2 & Exina & Sexina & Nexina 1 & Nexina 2 \\
\hline C. affinis & $6-(8)$ & 7,3 & 5,3 & 1,3 & 7,4 & 2,2 & 4,3 & 1,0 & 4,5 & 1,0 & 2,4 & 1,1 \\
\hline C. axillaris & 6 & 7,5 & 6,9 & 1,8 & - & - & - & - & 5,1 & 0,6 & 3,5 & 0,9 \\
\hline C. elongata & 6 & 7,9 & 6,4 & 1,4 & 9,3 & 4,1 & 3,8 & 1,3 & 7,5 & 3,0 & 3,2 & 1,3 \\
\hline C. ericoides & $6-(7-8)$ & 6,0 & 4,6 & 1,4 & 6,8 & 2,4 & 3,2 & 1,1 & 5,0 & 1,6 & 2,4 & 1,0 \\
\hline C. hirsuta & 6 & 8,0 & 6,9 & 2,0 & 7,0 & 2,8 & 3,1 & 1,1 & 7,0 & 2,8 & 3,1 & 1,1 \\
\hline C. linearifolia & $6-(7-8)$ & 5,6 & 4,4 & 1,4 & 8,7 & 2,9 & 4,6 & 1,1 & 6,9 & 2,1 & 3,6 & 1,1 \\
\hline C. sericea & $6(-7)$ & 4,6 & 3,6 & 1,3 & 6,6 & 2,5 & 2,9 & 1,1 & 5,9 & 2,1 & 2,7 & 1,1 \\
\hline C. affinis $\mathrm{x}$ & $6-(8)$ & 8,8 & 6,7 & 1,4 & 8,6 & 3,9 & 3,5 & 1,2 & 6,1 & 2,3 & 2,7 & 1,2 \\
\hline C. hirsuta & & & & & & & & & & & & \\
\hline
\end{tabular}

* $\mathrm{C}=$ comprimento $\mathrm{L}=$ largura $\mathrm{M}=$ margem 
gulares com a parte central ligeiramente côncava e ornamentada.

As medidas dos diâmetros e demais caracteres quantitativos encontram-se nas tabelas 1 e 2, respectivamente. Na tabela 1 nota-se, pelos valores do intervalo de confiança (IC) a 95\%, que os grãos de pólen de $C$. sericea destacam-se por apresentarem diâmetros significativamente menores em relação aos das demais espécies. A tabela 3 apresenta os dados dos materiais de comparação onde observa-se que as medidas dos diâmetros dos grãos de pólen não se mantém constantes entre os espécimes.

Chave polínica para identificação de espécies de Camarea:

1. Grãos de pólen com arestas.

2. arestas contínuas.

3. exina microrreticulada entre os colpóides

C. axillaris

3. exina levemente rugulada entre os colpóides C. ericoides, $C$. linearifolia

2. arestas descontínuas C. elongata

1. Grãos de pólen sem arestas.

4. poros pequenos $(<5,0 \mu \mathrm{m}$ compr.), exina psilada entre os colpóides

C. sericea

4. poros grandes $(>7,0 \mu \mathrm{m}$ compr.), exina microrreticulada entre os colpóides

5. colpóides finos ( $\overline{\mathrm{x}}=1,8 \mu \mathrm{m}$ larg. $)$, não ornamentados

C. affinis

5. colpóides largos $(\overline{\mathrm{x}}=7,3 \mu \mathrm{m}$ a $8,5 \mu \mathrm{m}$ larg. $)$ ornamentados

C. hirsuta, C. affinis x C. hirsuta

\section{Discussão}

Na revisão taxonômica que fez para o gênero Camarea, Mamede (1990a) considerou como espécies próximas: $C$. ericoides de $C$. linearifolia, $C$. sericea de $C$. hirsuta e, como muito próximas, $C$. elongata de C. axillaris e C. affinis de C. hirsuta.

Tabela 3. Medidas do diâmetro dos grãos de pólen dos materiais de comparação de Camarea.

\begin{tabular}{lc}
\hline Espécies & $\mathrm{x}(\mu \mathrm{m})$ \\
\hline C. affinis & \\
$\quad$ A.C. Brade 5315 & 64,0 \\
W. Handro 38 & 64,9 \\
O. Handro 64 & 64,6 \\
$\quad$ A.P. Duarte 5158 & 66,4 \\
C. affinis x C. hirsuta & \\
$\quad$ W.R. Anderson 6849 & 70,4 \\
C. axillaris & \\
$\quad$ M. Amélia V. da Cruz 87 & 60,7 \\
C. elongata & \\
$\quad$ G. Martinelli et al. 5244 & 65,1 \\
C. ericoides & \\
$\quad$ A. Macedo 3595 & 58,8 \\
$\quad$ H.S. Irwin et al. 9919 & 61,5 \\
$\quad$ L.O. William \& Vicente Assis 7571 & 61,2 \\
$\quad$ Mello Barreto 9821 & 58,8 \\
\hline
\end{tabular}

Os dados polínicos confirmaram esta proximidade. No caso de $C$. ericoides e $C$. linearifolia os grãos de pólen são similares, com exina levemente rugulada e providos de arestas salientes, lisas e contínuas, delimitando faces quadrangulares ligeiramente côncavas e ornadas (figuras 10 e 15). Embora tenham sido verificadas diferenças no tamanho do pólen, significativamente menor em $C$. linearifolia (tabela 1), este caráter não foi considerado de valor diagnóstico devido às diferenças encontradas nos grãos de pólen provenientes de diferentes espécimes de $C$. ericoides (tabelas 1 e 3 ), enquanto de $C$. linearifolia estudou-se o pólen de um único exemplar faltando parâmetros para comparação.

Camarea elongata e C. axillaris têm grãos de pólen microrreticulados, ornados por arestas salientes; a presença de arestas salientes caracteriza um grupo morfopolínico distinto dentro de Camarea formado por C. axillaris, C. elongata, C. ericoides e $C$. linearifolia. Arestas salientes, lisas e contínuas caracterizam o pólen de $C$. axillaris, enquanto em C. elongata estas são salientes e descontínuas. Makino-Watanabe (1988) estudou os grãos de pólen de C. triphylla Adr. Juss. (Martinelli et al. 5244 e Harley 19361) cujas exsicatas foram identificadas por Mamede (1990a) como sendo C. elongata, uma nova espécie do Morro do Chapéu, Bahia; neste trabalho a autora propôs a sinonimização de $C$. triphylla sob C. axillaris. 


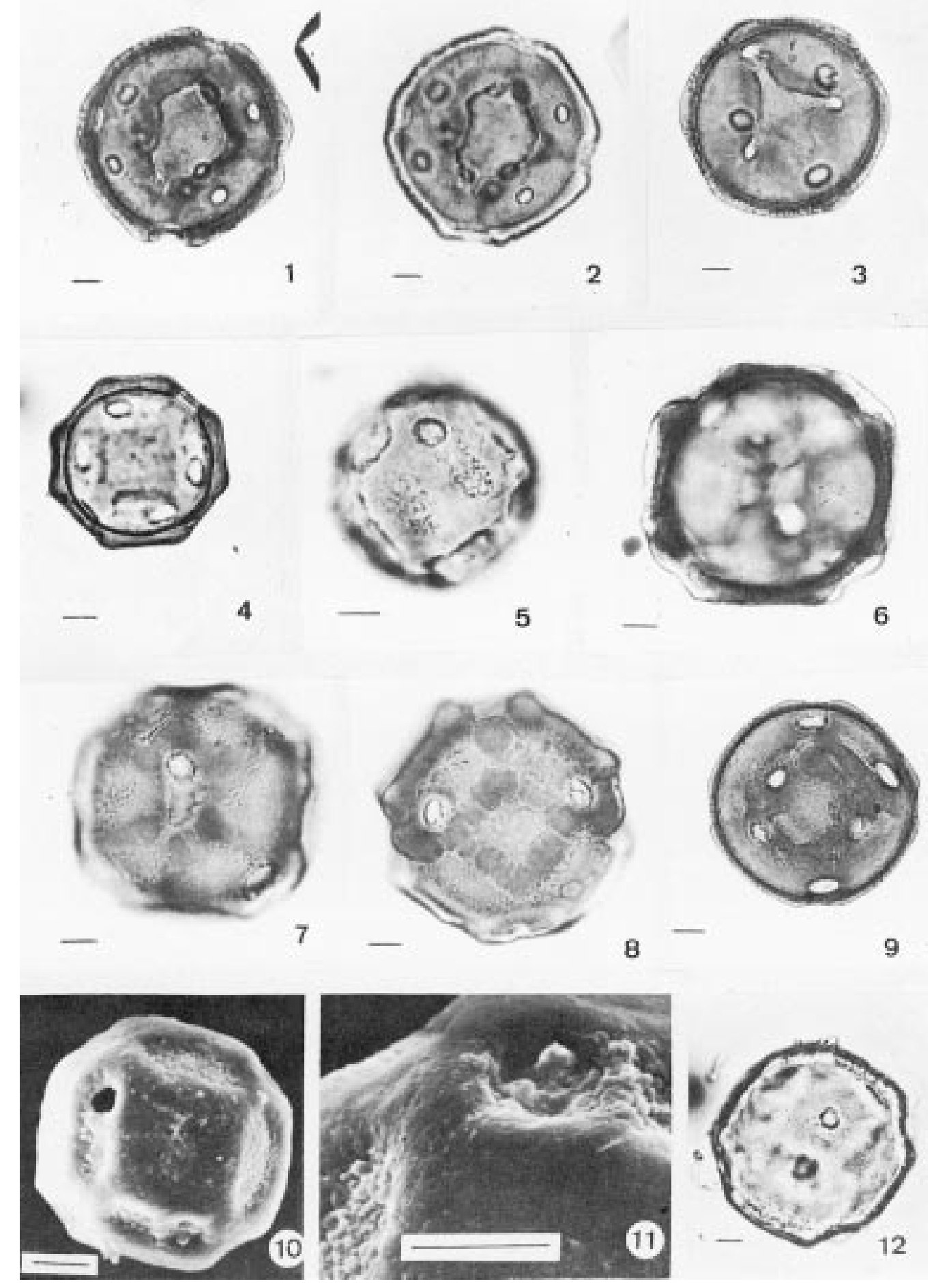

Figuras 1-12. Grãos de pólen de espécies de Camarea. 1-3. C. affinis. 1. Corte óptico da exina; 2. Superfície; 3. Colpóides. 4-5. C. axillaris. 4. Corte óptico; 5. Detalhe das aberturas e da ornamentação. 6-8. C. elongata. 6. Corte óptico; 7. Detalhe de um colpóide; 8. Detalhe de uma face quadrangular delimitada por arestas salientes descontínuas. 9-11. C. ericoides. 9. Corte óptico; 10-11. Elétronmicrografias. 10. Superfície do pólen; 11. Detalhe do poro e colpóides. 12. C. hirsuta; superfície. Escala = $10 \mu \mathrm{m}$. 

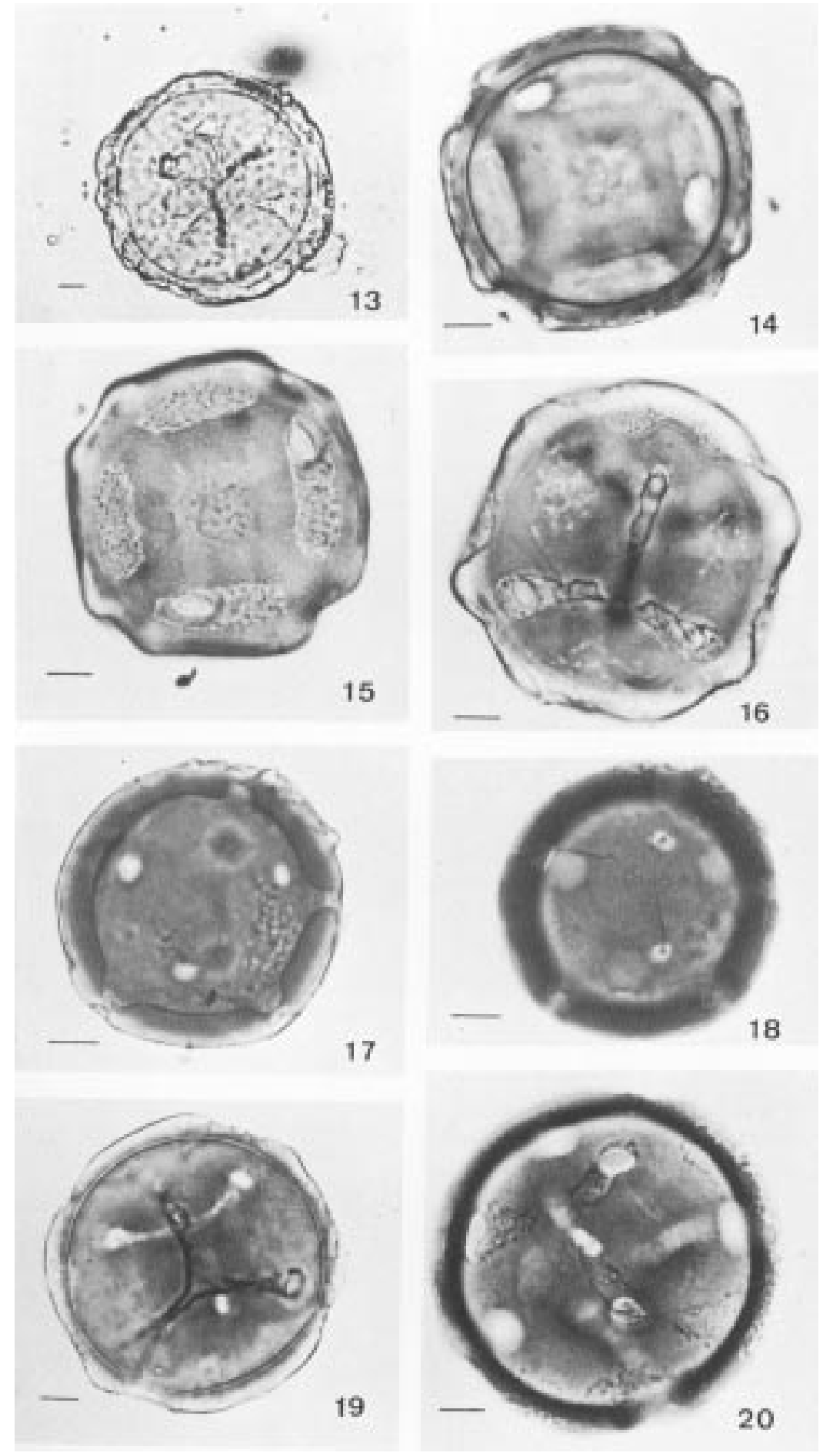

Figuras 13-20. Grãos de pólen de espécies de Camarea. 13. C. hirsuta; aberturas. 14-16. C. linearifolia. 14. Corte óptico; 15. Detalhe das aberturas e de uma das faces; 16. Colpóides; 17-18. C. sericea. 17. Corte óptico; 18. Poros e colpóides. 19-20. C. affinis x C. hirsuta. 19. Corte óptico; 20. Detalhe dos colpóides. Escala $=10 \mu \mathrm{m}$. 
Camarea sericea e C. hirsuta, do ponto de vista palinológico, estão incluídas no grupo de espécies onde o pólen é desprovido de arestas salientes, juntamente com $C$. affinis e seu provável híbrido com $C$. hirsuta. No caso de $C$. sericea e $C$. hirsuta, espécies taxonomicamente próximas, os grãos de pólen nos dois táxons são distintos quanto à ornamentação da exina e ao tamanho do poro. Em $C$. sericea os grãos de pólen são psilados, com poros pequenos (ca. 4,6 x 3,6 $\mu \mathrm{m}$ ) enquanto em $C$. hirsuta são microrreticulados com poros grandes (ca. $8,0 \times 6,9 \mu \mathrm{m}$ ).

Através do exame do isótipo de Camarea glazioviana, Mamede (1990a) constatou que o material correspondia na realidade a $C$. sericea e propôs a sinonimização desta sob a primeira. Assim, o espécime de C. glazioviana (P. Gibbs et al. 2763), cujo pólen foi estudado por Makino-Watanabe (1988), é, na realidade, C. sericea.

Camarea affinis e $C$. hirsuta são duas espécies muito próximas, inclusive do ponto de vista palinológico, porém distintas pelo tipo de abertura dos grãos de pólen, ou seja, colpóides finos, não esculturados em C. affinis e largos, esculturados em C. hirsuta.

Dos materiais de Camarea hirsuta referidos por Makino-Watanabe (1988), apenas a coleta, Schwacke 3305, permaneceu como tal; os demais (Anderson 6849 e Anderson 7948) foram considerados como prováveis híbridos entre $C$. affinis e $C$. hirsuta (Mamede 1990a).

Sabe-se que o pólen pode ser usado nos estudos com híbridos permitindo inclusive avaliar as características dos ancestrais (Erdtman 1969). Estudos anatômicos e palinológicos realizados por Mamede (1990b e 1993) suportam a condição intermediária deste provável híbrido. Quanto ao padrão de venação, a autora verificou que o do híbrido era semelhante ao de $C$. hirsuta. No presente estudo verificou-se que o pólen do híbrido está mais relacionado com o de $C$. hirsuta considerando-se o tipo de colpóide e o tamanho do poro. Mamede (1990b) não fez menção à presença de colpóides, estrutura também não mencionada por Erdtman (1971) para o pólen de C. affinis e C. axillaris (sob C. triphylla) e por Salgado-Labouriau (1973), para o tipo de Camarea.

Os resultados obtidos mostram que no gênero Camarea é possível a separação das espécies baseada em caracteres morfológicos dos grãos de pólen. Estes dados reforçam os posicionamentos apresentados na revisão taxonômica deste táxon, feita por Mamede (1990a).

Agradecimentos - Ao Agente de Apoio à Pesquisa Científica e Tecnológica, Sr. Elvis Alves de Godoy, pela digitação do trabalho.

\section{Referências bibliográficas}

ERDTMAN, G. 1960. The acetolysis method. A revised description. Svensk. bot. Tidskr. 54:561-564.

ERDTMAN, G. 1969. Handbook of palynology. Hafner Publ. Comp., New York.

ERDTMAN, G. 1971. Pollen morphology and plant taxonomyAngiosperms. Hafner Publ. Comp., New York.

MAKINO-WATANABE, H. 1988. Contribuição ao estudo palinológico das Malpighiaceae A. L. Jussieu do Brasil (Tribo Banisterieae, subtribo Banisteriinae). Tese de doutorado, Universidade Estadual de Campinas, Campinas.

MAMEDE, M. C. H. 1990a. Revisão do gênero Camarea SaintHilaire (Malpighiaceae). Hoehnea 17:1-34.

MAMEDE, M. C. H. 1990b. Observações sobre a ocorrência de prováveis híbridos entre Camarea affinis St.-Hil. e Camarea hirsuta St.-Hil. (Malpighiaceae). Hoehnea 17:35-46

MAMEDE, M. C. H. 1993 Anatomia dos orgãos vegetativos de Camarea (Malpighiaceae). Acta bot. bras. 7:3-19.

SALGADO-LABOURIAU, M. L. 1973. Contribuição à palinologia dos cerrados. Acad. Bras. Ciênc., Rio de Janeiro. 